\title{
Aging, rhythms of physical performance, and adjustment to changes in the sleep-activity cycle
}

\author{
T Reilly, J Waterhouse, G Atkinson
}

\begin{abstract}
Objectives-Shiftwork causes disturbances of the normal sleep-wake cycle and circadian rhythm. There is concern that aging workers have more problems than younger counterparts when the human body clock is disrupted. This review considers issues relating to aging, the circadian body clock, and adjustment to altered sleep-wake schedules.

Methods-Reports on effects of aging on the human body clock were reviewed. Research concerned with adjustment to circadian phase shifts (as occurs in night work) was considered.

Results-With aging there is an increased tendency towards morningness which is linked with difficulties in sleeping. The peak time and amplitude of normal circadian rhythms are altered. Tolerance of shiftwork can be linked with social factors as well as adaptation of the body clock.

Conclusions-People habituated to night work seem to have developed mechanisms which allow them to cope with disruptions to lifestyle and the endogenous body clock. Elderly people are more suited to phase advances, as occur in morning workshifts, than to phase delays such as nocturnal work.
\end{abstract}

(Occup Environ Med 1997;54:812-816)

Keywords: circadian rhythms; night work; sleep disturbance

Many industrial and service operations, due to their social or economic importance, have to be sustained over the 24 hour day. Consequently, a considerable proportion of the national workforce is periodically engaged in shiftwork to support the occupations associated with these operations. The rate of participation in shiftwork has been estimated to be $10 \%-25 \%$ of all those employed, with higher rates among British male manual workers and Americans employed in part time work. ${ }^{12}$ It has been estimated that $17.6 \%$ of European workers are engaged in night work for at least a quarter of their working time and about 20 million full time workers in the United States are involved in shiftwork. ${ }^{3}$

Shiftwork has been defined for purposes of clarity as "any regularly taken employment outside the day working window, defined arbitrarily as the hours between 0700 and 1800 hours". ${ }^{4}$ This definition incorporates schedules that are fixed to nocturnal or part nocturnal times of day, and schemes whereby the shift is rotated to allow the employee to return regularly to a diurnal schedule. Even so, participation in shiftwork is likely to induce strain from social and domestic sources, sleep problems, and disturbances to the circadian body clock. ${ }^{56}$ The participation in shiftwork spans a wide range of ages. It is relevant to consider the effects of age on the human body clock, the extent to which aging affects tolerance to disturbances of the clock, and what behavioural strategies are adopted to tolerate shiftwork.

The human body clock is regulated endogenously by the suprachiasmatic nuclei in the hypothalamus. ${ }^{7}$ Under nychthemeral conditions many biological functions, illustrated by the curve in rectal temperature (fig 1) follow a cyclical pattern. This is closely related to the rhythm in human physical performance, as indicated by self chosen workrate (fig 2): very many human performance measures (both physical and mental) follow this pattern, reaching a nadir in the early hours of the morning. ${ }^{89}$ This is reflected in the occurrence of mistakes made in a whole range of tasks as well as catastrophic events implicating human error at night. ${ }^{2}$

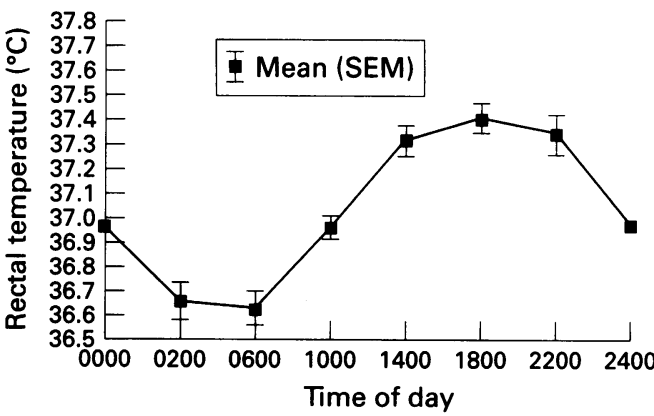

Figure 1 The normal circadian curve (mean (SEM)) in body temperature in men aged 21-37.

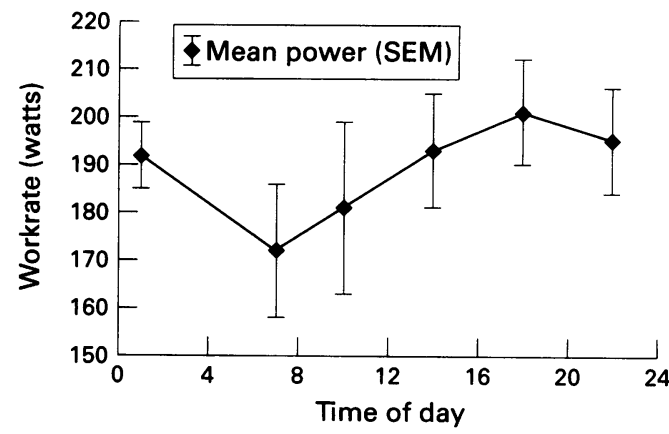

Figure 2 The circadian rhythm in exercise performance shown by the mean power output (W) in a self paced ergometer cycling test. Subjects were 13 men, aged 19-27. 
With continued night work, circadian rhythms begin to adjust to the altered sleepwake cycle. Assessment of how much adjustment has taken place is complicated by the fact that the measured rhythm reflects the sleepwake cycle as well as the body clock: therefore during night work the altered sleep-wake cycle will lead to the apparent phase change of a circadian rhythm being an exaggeration of the shift of the body clock. ${ }^{10}$ However, examination of the circadian rhythms of shiftworkers indicates that even those on permanent night work do not show a complete adjustment of the body clock to their altered lifestyle. ${ }^{11}$ The disruption of sleep (duration and quality) may be accentuated in aging workers as sleep patterns are affected by age. Alterations in meal times can lead to gastrointestinal problems, the persistence of which leads to a drop out from shiftwork at an early age. Disruption of leisure activities is also a major factor in drop out.

The concern in this overview is with human circadian rhythms and tolerance to disturbances in circadian rhythms due to shiftwork and travel across multiple time zones. The broad perspective is to examine how circadian rhythm characteristics change with age and establish whether these changes are related to tolerance to, and efficiency on, unusual work and travel schedules. This research review will concentrate on recent findings from our group in Liverpool and place the findings in a broader context. The specific issues within the review are: (a) to establish the effects of age on selected human physiological and performance rhythms; $(b)$ to determine any reductions with aging in the ability to cope with phase shifts.

\section{Effects of age on the normal circadian} rhythm

Sleep patterns are affected by aging. Infants under the age of 2 years generally sleep for about $60 \%$ of the solar day. From then on there is a reduction in hours slept, the afternoon nap generally being abandoned at about the age of four. ${ }^{12}$ During the more productive years of adulthood the mean hours slept in each solar day are about seven. This mean value hides a large variability in the hours of sleep taken, although this may not correspond with individual need.

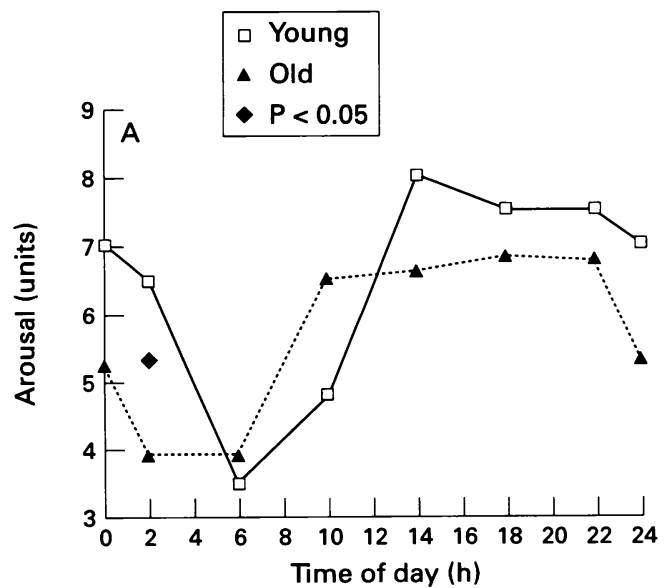

With aging there is increased difficulty in getting to sleep and generally a shorter nocturnal sleep. ${ }^{13}$ Earlier waking may be compensated for by an increased tendency to nap in the afternoon for retired people or after work for those nearing retirement age. The earlier waking is consistent with an increased morningness in aging people. ${ }^{14}$ The dip in arousal after lunch found in subjects of all ages ${ }^{7}$ implies that mental fatigue is more likely to occur at this time in older workers who might be slightly sleep deprived. The tendency to nap later in the day coincides with a subharmonic in the circadian rhythms of aging people.

Both of these phenomena have also been identified in a study of hypertensive people. ${ }^{15}$ Different age groups of hypertensive people were examined for circadian rhythms in blood pressure and heart rate. These were determined by means of ambulatory devices and analysed for ultradian (cycles $<24$ hours) components. Thirty one hypertensive patients consisting of young ( $n=9$, aged 25-44), middle aged $(n=11$, aged $47-57)$ and old $(n=11$, aged 57-74) groups acted as subjects. Measurements were made in a hospital ward after a drug free run-in period. Blood pressure and heart rate were recorded automatically every 20 minutes during the day and every 30 minutes at night. The amplitudes of rhythms in systolic blood pressure and heart rate were found to be reduced with age. The reductions in amplitude were largely attributable to increases in night time values for the older subjects. The older hypertensive patients also showed an unusual blood pressure profile, with a postlunch hypotension and a secondary evening peak. There was also a change with age in the phase relation between systolic blood pressure and heart rate. This suggests an internal dissociation of rhythms with aging.

Subjective states also showed a circadian rhythm, particularly those predisposing towards work performance. The effect of age on subjective arousal (fig 3A) and vigour showed the characteristic earlier acrophase (peak) and lowered amplitude in older subjects than in younger people. ${ }^{16}$ The acrophase in sleepiness was found to be more variable in the older subjects, who showed rhythms in fewer moods

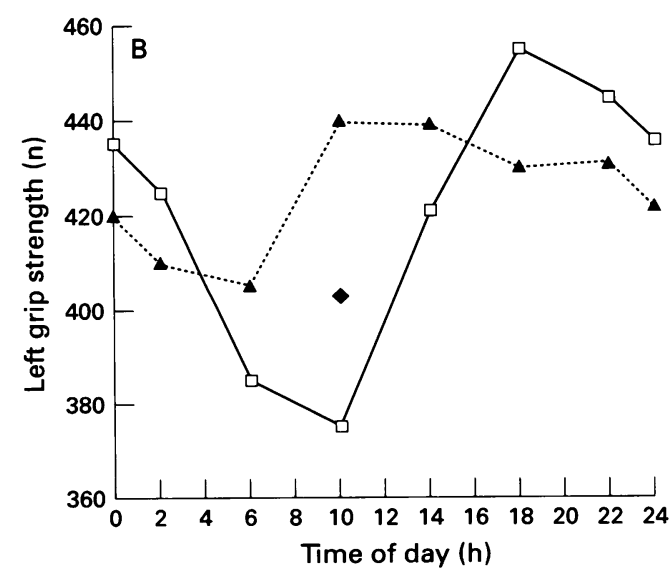

Figure 3 Subjective arousal $(A)$ and left grip strength (B) at different times of the day in a young (aged 18-29) and an old (aged 47-62) experimental group. 
than did the younger group. Time estimation did not vary with the time of day in the older subjects whereas its rhythm conformed to that of body temperature in the younger people.

The major changes in circadian rhythms that occur with aging are a shift towards morningness (that is, a phase advance) and a reduction in amplitude ${ }^{1314}$ which leads towards a truncated rise during the daytime. There is also the tendency towards a bimodal shape to the circadian curve in some variables and more variability in the acrophase between people. ${ }^{17}$ Although these changes are apparent in rhythms of subjects at rest, the next question is whether the same trends are evident in human performance measures or in responses to activity.

\section{Aging and human performance rhythms} Circadian rhythms relevant to physical activity were investigated by Atkinson $e t a l^{18}$ in different age groups. Subjects were divided into a young (mean age 24, range 18-29) and an old (mean age 51, range 47-62) group. All subjects were physically active and regularly participated in sport to standardise for fitness levels. A comprehensive test battery was given to the subjects at $0200,0600,1000,1400,1800$, and 2200 hours in a counterbalanced design. At least eight hours separated each test session to allow for recovery. Familiarisation, pretest conditions, control of sleep, environmental conditions, etc all conformed to the requirements of research design in chronobiological studies. ${ }^{2}$

Rhythm acrophases derived from cosinor analysis showed the earlier acrophases in the old compared with the young group (fig 4). This applied to physiological markers (body temperature), indices of wakefulness (arousal), muscle strength, dynamic muscular performance (flight time in a vertical jump test) and the self chosen work intensity (measured as power output in a self paced ergometer task). The acrophase for the six work performance measures occurred 2.83 (SD 1.48) hours earlier for the old group than for the young group $(P<0.01)$.

In this study the amplitude of the circadian rhythms was significantly reduced for rectal

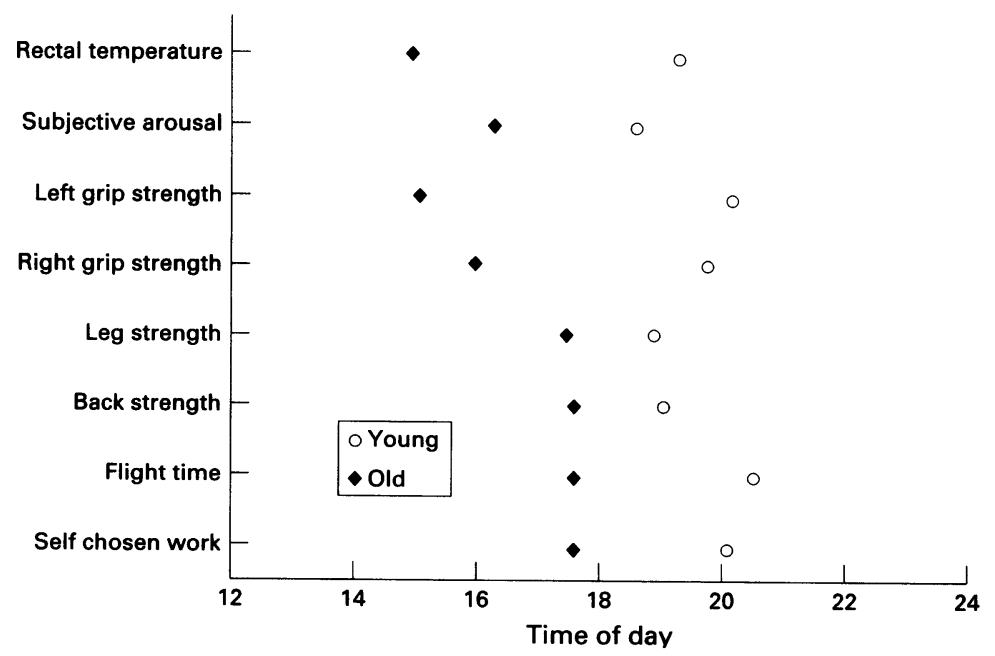

Figure 4 Separation of young (18-29) and old (47-62) workers on the basis of acrophases in a range of circadian rhythms. temperature, resting pulse rate, and left grip strength (fig 3B) in the older group. ${ }^{18}$ Although other rhythm amplitudes (such as arousal) were lower in the old subjects compared with the young group, they did not reach significance. This was attributed to the high physical fitness of the subjects recruited for the study. Atkinson et $a l^{19}$ reported that rhythm amplitudes are greater in subjects who carry out high levels of habitual physical activity and who are deemed physically fit. Indeed, well trained older subjects showed rhythm amplitudes that were higher than those of an inactive, unfit younger group. It was suggested that the maintenance of physical activity in old age is of benefit in prolonging efficient control of circadian rhythms.

Performance in many dynamic occupational tasks is influenced by range of motion or flexibility as well as muscular strength. The typical circadian variation in spinal flexibility found in young people was not found in an old group. ${ }^{16}$ This may mean that older workers have lower tolerance for tasks that load the spine. Spinal shrinkage has been used as a tool to indicate loading on the spine, is responsive to a range of occupational tasks and shows a characteristic diurnal variation of about $1 \%$ of stature. ${ }^{20}$ Although studies of shrinkage support an increased vulnerability with aging of the intervertebral discs in instances of spinal loading, the occupational implications have not been fully investigated.

Further insights into capabilities of work performance can be obtained from studies of competitive cyclists. Veteran cyclists seem more likely to be morning types, as they schedule greater amounts of their training before 1400 hours than do young adults. This is evident before the older cyclists have retired from work. ${ }^{21}$ The performance of veteran cyclists (aged $\geqslant 40$ ) in time trials is also less affected by early morning starts. Although subjects aged 50-60 years still perform best in the early evening, they also perform relatively well in the morning, with age differences in performance being least at this time, ${ }^{22}$ notwithstanding that, in general, veterans' performances are worse than those of younger subjects.

It is not clear whether such findings reflect age related changes in the endogenous clock or exogenous influences such as the timing of sleep or other aspects of lifestyle. There is evidence for both, ${ }^{13} 142324$ and they would normally be linked and so change together. Aging is associated with a large variation between people in the loss of mental and physical powers, as well as robustness of circadian rhythms. Old non-institutionalised people studied by Minors $e t a l^{25}$ showed less day to day variations in their lifestyles as they aged, indicating that they had become more set in their ways. It was concluded that a deteriorating body clock contributes to some of the changes in lifestyle, but that the development by a person of an increasingly inflexible lifestyle offsets some of the effects on the decline in circadian rhythmicity. Such independence becomes increasingly unrepresentative of old subjects as a group; these people have been called 
"survivors". ${ }^{24}$ Those who become institutionalised, often with decreased physical powers, become ever more dependent on the imposed routine for maintenance of circadian rhythmicity.

Normally, physical performance corresponds best with the rhythm in body temperature whereas most measures of mental performance tend to be best at the high point of the diurnal rhythm in arousal. ${ }^{8}$ Physical performance that has to be sustained at a high intensity may lead to fatigue due to reduction in energy stores in the active muscles or an increase in body temperature beyond the optimal level. In such prolonged tasks the performance is more evenly paced in the morning; when the lower self chosen work rate in the morning is better maintained than the initial higher load selected in the evening. ${ }^{26}$ Although fatigue (as indicated by a fall in work rate) associated with prolonged tasks is inevitable, it is least in older subjects when the task is performed in the morning (fig 5 ). ${ }^{27}$

\section{Night work}

There is a general view that increasing age is associated with decreased tolerance of shiftwork although the reasons for this are poorly understood. ${ }^{5}$ A general decrease in physiological adaptability has been linked to the reduction in rhythm amplitude that occurs with age. ${ }^{27}$ The earlier acrophase that occurs with aging is disadvantageous for night work but could be an advantage for the morning shift and for getting to sleep early enough during the evening before. In the United States it is common for older and more senior shiftworkers to be on the morning shift and night work is performed mainly by younger members of the workforce.

Tolerance of shiftwork can also be influenced by social factors. ${ }^{28}$ Middle aged people with a family that has grown up and with decreasing

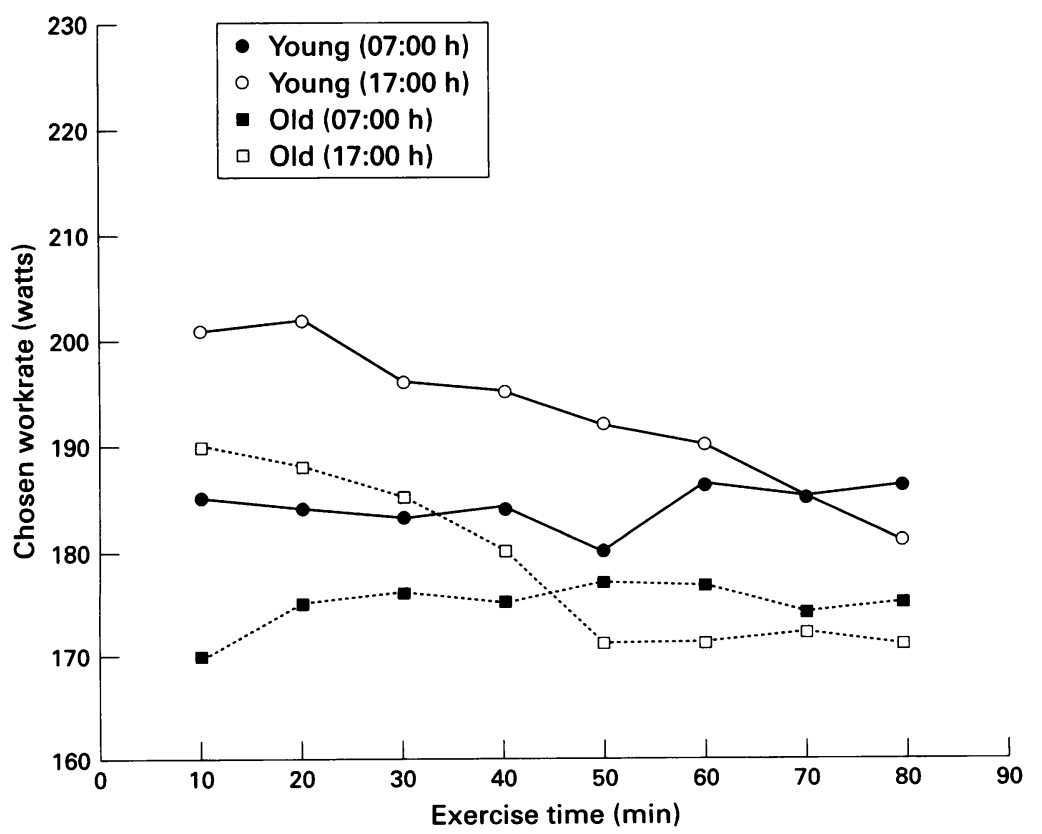

Figure 5 Self paced work performance in a prolonged ( 80 minute) physical activity task in the morning and evening for young (19-25) and old (48-62) groups. In both groups, the interaction between time of day and duration of exercise was significant $(P<0.05)$. financial commitments may be less motivated by financial and promotional rewards to offset the unsocial hours that they work than was the case earlier in their career. The dislike of shiftwork by younger workers may be dominated by social factors (such as leisure or family activities) rather than physiological intolerance. In a study of 163 nurses on nocturnal regimens ${ }^{16}$ it was found that: (a) shiftwork was more acceptable to a middle aged (mean (SD) 34 (3)) group than to a young (22 (1)) or old (49 (5)) group, and was related to the number of family dependants; $(b)$ the older nurses showed a lower flexibility in their sleeping habits as well as increased morningness; $(c)$ the workers' coping strategies were unaffected by age or experience of shiftwork.

Reilly et $a l^{16}$ also investigated age related responses in selected physiological and performance measures after a phase shift in young (27 (2)) and old (45 (5)) security workers and car manufacturers. All worked a backward rotating shift (night 2200 to 0600 , afternoon 1400 to 2200 , and morning 0600 to 1400 ) which required a phase advance of their circadian rhythms. The car manufacturers worked a slow rotating system, comprising five work periods a week then two rest days, whereas the security workers also operated on a slow rotating shift but with seven work periods then two to three rest days. Measures taken every two hours included body temperature, grip strength, lung function, and subjective arousal. Results confirmed that the circadian rhythms in the older group were more disturbed (greater alterations in their rhythm characteristics) and the older subjects were less capable in the performance measures. This was by contrast with the results at the start of the morning shift when the differences between the two age groups were smallest at 0600 hours, the subjects having slept during the preceding night (in preparation for starting the morning shift). The early end of sleep (to start work at 0600 hours) had a large negative influence on the younger workers whereas for the older workers the early start was less disruptive. This finding is compatible with the conclusion that older people are better suited to working morning rather than night time shifts. ${ }^{9} 1314$

Shiftwork has been linked with an increased incidence of cardiovascular disease ${ }^{52}$ and digestive disorders. ${ }^{50}$ The main stresses seem to be the disruption of circadian rhythms, accumulated disturbances of sleep, and chronic fatigue. It seems that there are health problems for shiftworkers independent of the effects of aging. Older workers who tolerate shiftwork systems seem to have been highly self selective as those intolerant may have dropped out after early exposure. ${ }^{50}$

\section{Travel fatigue}

The travel fatigue associated with long haul flights is due to disruption of the body's circadian clock when multiple time zones are crossed. The syndrome known as jet lag is not experienced in flights within the same time zone. Despite their greater experience of air travel, older people tend to experience more 
severe jet lag symptoms than younger travellers. ${ }^{31}{ }^{32}$ Symptoms also tend to last longer the older the person. It is possible that the lower amplitudes of their physiological rhythms and their more sedentary lifestyle place elderly people at a disadvantage but this has not been confirmed. It is unclear if the poorer adjustment is indicative of an inability to alter lifestyle, the body clock, or a combination of both.

As older people tend to be responsible for crucial business decisions after long haul flights, it is recommended that important meetings are scheduled for hours of day when jet lag is likely to be minimised or the traveller has had time to adjust to the new time zone. The body clock adjusts more easily to a phase delay than to a phase advance and so jet lag is not so severe on travelling westwards compared with eastwards. It is possible that older people are less disadvantaged on travelling eastwards because of their earlier acrophase.

\section{Overview}

There is evidence that the control of circadian rhythms in physiological and performance variables is affected by age. The extent to which this is due to nerve cell decay in the suprachiasmatic nuclei of the hypothalamus - the site of the body clock-has not been determined. Older subjects show a reduced amplitude and a tendency towards morningness in their circadian cycles. Pronounced effects of age are not evident in the rhythms of workers habituated to nocturnal shift systems due to their coping mechanisms. Even so, it seems that they are more suited chronobiologically to morning shifts and to phase advances than to night shift regimens.

1 Young BM. The shift towards shiftwork. New Society 1982;61:96-7.

2 Reilly T, Atkinson G, Waterhouse J. Biological rhythms and exercise. Oxford: Oxford University Press, 1997.

3 Costa G. The problem: shiftwork. Chronobiol Int 1997:14: 89-98.

4 Monk TH, Folkard S. Making shiftwork tolerable. Basingstoke: Taylor and Francis, 1992

5 Waterhouse J, Folkard S, Minors D. Shiftwork, health and Waterhouse J, Folkard S, Minors D. Shiftwork, health and
safety. An overview of the scientific literature 1978-90. safety. An overview of
London: HMSO, 1992 .

6 Rutenfranz J, Colquhoun W, Knauth P, Ghata N. Biomedical and psychosocial aspects of shift work. A review. Scand f Work Environ Health 1977;3:165-82.

7 Minors D, Waterhouse J. Circadian rhythms and the human. Bristol: John Wright, 1981

8 Reilly T. Human circadian rhythms and exercise. Crit Rev Biomed Eng 1990;18:165-80.

9 Folkard S. Circadian performance rhythms: some practical and theoretical implications. Philos Trans $R$ Soc Lond B Biol Med 1990;327:543-53.
10 Minors D, Waterhouse J. Separating the endogenous and exogenous components of the circadian rhythm of body temperature during night work using some "purification models. Ergonomics 1993;36:497-507.

11 Patkai P, Akerstedt T, Pettersson K. Field studies of shift work: I. Temporal patterns in psychophysiological activation in permanent night workers. Ergonomics 1977;20:611-

12 Hellbrugge T. The development of circadian and ultradian rhythms of premature and full-term infants. In: L Scheving F Halberg, J Pauly, eds. Chronobiology. Tokyo: Igaku Shoin, 1974:339-41.

13 Kerkhof G. Circadian rhythms in old age. Acta Physiologica Polonica 1988;39:357-63.

14 Monk T. Circadian rhythm. Clin Geriatr Med 1989;5:33146.

15 Atkinson G, Witte K, Nold G, Sasse U, Lemmer B. Effects of age on circadian blood pressure and heart rate rhythms of age on circadian blood pressure and heart rate rhythms in patients

16 Reilly T, Atkinson G, Coldwells A. Ageing and shiftwork. Liverpool: John Moores University, Report to the Health and Safety Executive, 1993.

17 Waterhouse J, Minors D. Circadian rhythms and aging. Reviews of Clinical Gerontology 1995;5:369-78.

18 Atkinson G, Coldwells A, Reilly T, Waterhouse JM. An agecomparison of circadian rhythms in physical performance measures. In: Harris S, Suominen H, Era P, Harris WS, eds. Towards healthy aging: international perspectives part 1 eds. Towards healthy aging: international perspectives part 1 . Physiological and biomedical aspects. Vol 3: physical activity, aging and sports.

19 Atkinson G, Coldwells A, Reilly T, Waterhouse J. A comparison of circadian rhythms in work performance between physically active and inactive subjects. Ergonomics 1993;36:273-81

20 Reilly T, Boocock MG, Garbutt G, Troup JDG, Linge K Changes in stature during exercise and sports training. Applied Ergonomics 1991:22:308-11.

21 Atkinson G, Coldwells A, Reilly T, Waterhouse J. Effects of age on diurnal variations in prolonged physical performage on diurnal variations in prolonged physical performSports Sciences 1994;12:127.

22 Atkinson G, Coldwells A, Reilly T, Waterhouse J. An age comparison of circadian rhythms in physical performance and mood states. fournal of Interdisciplinary Cycle Research 1992;23:186-8.

23 Czeisler C, Dumont M, Duffy J, Steinberg J, Richardson G Brown $\mathrm{E}$, et al. Association of sleep-wake habits in olde people with changes in output of circadian pacemaker. Lancet 1992;340:933-6.

24 Monk T, Reynolds C, Buysse D, Hoch C, Jarrett D, Jennings $\mathrm{J}$, et al. Circadian characterisitics of healthy 80 -year-olds and their relationship to objectively recorded sleep. $f$ Gerontol A Biol Sci Med Sci 1991:46:M171-5.

25 Minors D, Atkinson G, Bent N, Rabbitt P, Waterhouse J

The effects of age upon some aspects of lifestyle and implications for studies on circadian rhythmicity. Age Research cations for studies
1997 (in press).

26 Reilly T, Garrett R. Effects of time of day on self-paced performance of prolonged exercise. I Sports Med Phys Fitness 1995;35:99-102.

27 Atkinson G, Reilly T. Effects of age and time of day on preferred work-rates during prolonged exercise. Chronobiol In 1995;12:121-9.

28 Walker J. Social problems of shiftwork. In: S Folkard, T Monk, eds. Hours of work. Chichester: Wiley, 1985:211-25.

29 Kristensen TS. Cardiovascular diseases and the work environment. A critical review of the epidemiologic environment. A critical review of the epidemiologic
literature on non chemical factors. Scand $f$ Work Environ literature on non chemical

30 Harrington JM. Shiftwork and health: a critical review of the literature. London: Her Majesty's Stationary Office, 1978.

31 Reilly T, Atkinson G, Budgett R. Effects of temazepam on physiological and performance variables following westerly flight across five time zones. Fournal of Sport Sciences 1997;15:62.

32 Monk T, Buysse D, Reynolds C, Kupfer D. Inducing jet lag in older people: adjusting to a 6-hour phase advance in routine. Exp Gerontol 1993;28:119-33. 